\title{
Sensor Data Gathering Time from Multiple Zero Standby power-type Sensor Nodes Simultaneously Awaken
}

\author{
Akira Yamawaki ${ }^{\mathrm{a},{ }^{*}}$, Makoto Miyauchi ${ }^{\mathrm{b}}$ and Seiich Serikawa ${ }^{\mathrm{a}}$ \\ ${ }^{a}$ Kyushu Institute of Technology, 1-1 Sensui, Tobata, Kitakyushu 804-8550, Japan \\ bNational Institute of Technology, Kitakyushu College, 5-20-1 Shii, Kokuraminami, Kitakyushu 802-0985, Japan \\ *Corresponding Author: yama@ecs.kyutech.ac.jp
}

\begin{abstract}
To realize an efficient infrastructure monitoring system, we have proposed combination of zero standby power-type sensor node (ZSSN) and UAV. The many ZSSNs with a battery are attached to a target infrastructure. They never consume any power from the battery when standby mode. So, the ZSSNs can reduce the burden replacing and charging many batteries. The UAV like a drone is gathering the sensed data from ZSSNs while traveling along the infrastructure waking the ZSSNs up. Since the ZSSNs wake up from the state power supply is completely cut, the waking time may be longer. As a result, the traveling time of UAV also becomes longer. This may lead to shorten the battery life of UAV and to enlarge the maintenance cost after all. This paper investigates the gathering time from multiple ZSSNs simultaneously awaken and discusses how much reduce the traveling cost of UAV by eliminating the necessity to visit all ZSSNs.
\end{abstract}

Keywords: Sensor network, low power, Gathering time.

\section{Introduction}

To realize an efficient infrastructure monitoring system, we have proposed combination of zero standby power-type sensor node (ZSSN) and UAV like drone ${ }^{(1-5)}$. In the proposed monitoring system, many ZSSNs with a battery are attached to a target infrastructure. The ZSSN cuts the ground line of power rail in standby mode. So, the standby power can be eliminated completely. The ZSSNs are waken up by the UAV irradiating a strong pulsed infrared light. The ZSSN has a photo diode generating an electromotive forth by receiving the strong pulsed infrared light. This electromotive forth connects the ground line and the ZSSN wakes up, senses the status of the infrastructure, and sends the sensed data to the UAV. The UAV automatically travels all ZSSNs attached to the infrastructure, waking them up and gathering the sensed data.

By using our system, the following burden caused by the infrastructure maintenance can be eliminated.

(1) Battery replacement for many sensor nodes.

(2) Battery charge for many sensor nodes.

(3) Gathering sensor data.

However, the ZSSN has a drawback compared with the conventional sensor node whose power is constantly supplied.

Since the ZSSNs wake up from the state power supply is completely cut, the waking time may be longer. As a result, the traveling time of UAV also becomes longer. This may lead to shorten the battery life of UAV and to enlarge the maintenance cost after all. This paper investigates the gathering time from multiple ZSSNs simultaneously awaken and discusses how much reduce the traveling cost of UAV by eliminating the necessity to visit all ZSSNs.

The rest of paper is organized as follow. Section 2 briefly explains the ZSSN and infrastructure monitoring system using ZSSN clarifying the research objective this paper targets. Section 3 shows the experimental setup to obtain the gathering time of sensed data from two ZSSNs. Section 4 shows the experimental results and discusses them. Finally, Section 5 concludes this paper.

\section{Zero Standby Power Type Sensor Node}

\subsection{ZSSN Architecture}

The zero standby power-type sensor node (ZSSN) attempts to greatly expand the battery life by completely eliminating the standby power consumption and using 


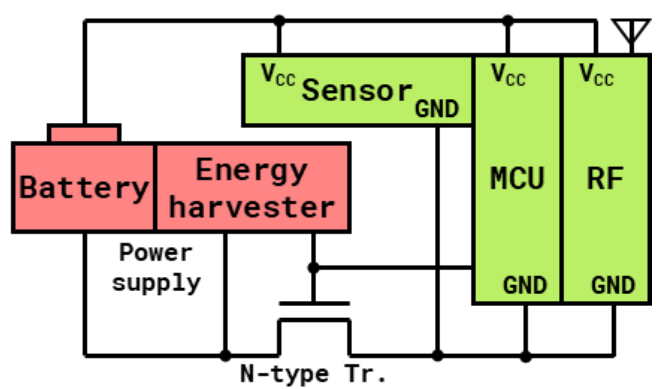

Fig. 1 Zero Standby power-type Sensor Node

adaptive wake/sleep scheduling. These promising features have never been seen on the conventional sensor nodes.

Fig.1 shows the architecture of ZSSN. The ZSSN has the unique power supply combining a battery and an energy harvester consisting of sensor. The energy harvester consists of some sensor generating electromotive forth by an even stimulated. Such kinds of sensors may be a photovoltaic, wind sensor, piezo element, peltier element, and so on. The ground line is bridged by an n-type power transistor. The ZSSN also has a sensor to investigate the status of the infrastructure, a microcontroller (MCU), and a radio frequency module (RF).

\subsection{ZSSN Action}

The ZSSN turns off the n-type transistor bridging the ground line in standby state. As a result, the standby power is never consumed by any modules on the ZSSN since there is no closed loop from battery to battery. We call the standby mode of the ZSSN as the apparent death mode. Fig.2 shows an overview of ZSSN actions. Fig.2 (a) corresponds to the apparent death mode.

When the energy harvester is stimulated by an event as shown in Fig.2 (b), it generates a weak electromotive forth. The responsibility of this small electromotive forth is only to turn on the n-type power transistor to connect the ground line at a moment. As a result, the microcontroller wakes up.

The microcontroller awaken is continue to turn on the n-type transistor instead of the energy harvester as shown in Fig.2 (c). As a result, the RF module is also awakened up and can establish the stable wireless network using the enough power from the battery. The microcontroller acquires the sensed data from the sensor and transmits it to the wireless network through the RF module. The ZSSN finishing the series of jobs turns off the n-type transistor. The ZSSN implicitly goes to the apparent death mode consuming no power.

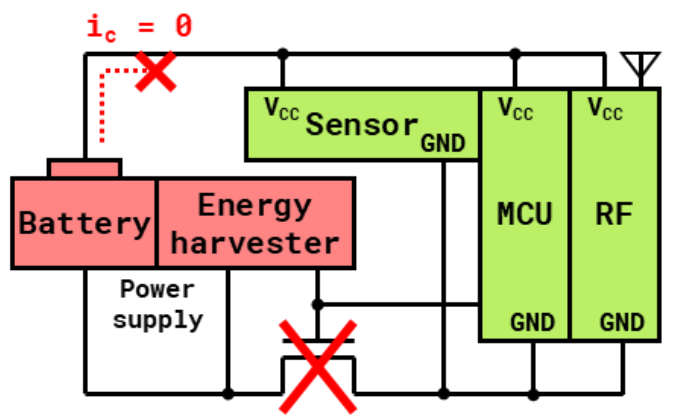

(a) Apparent death

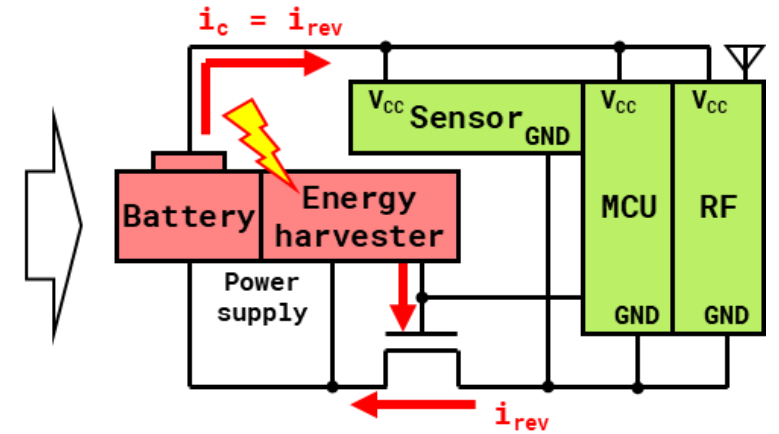

(b) Revival

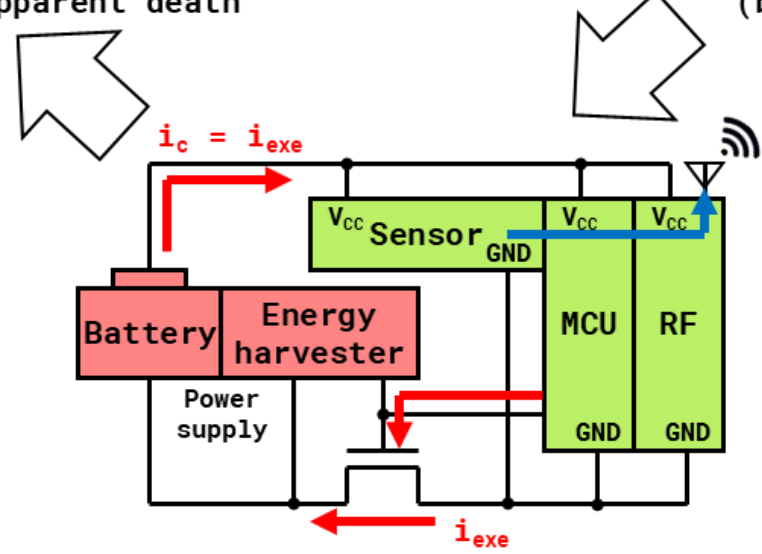

(c) Execution

Fig. 2 Overview of ZSSN Actions 

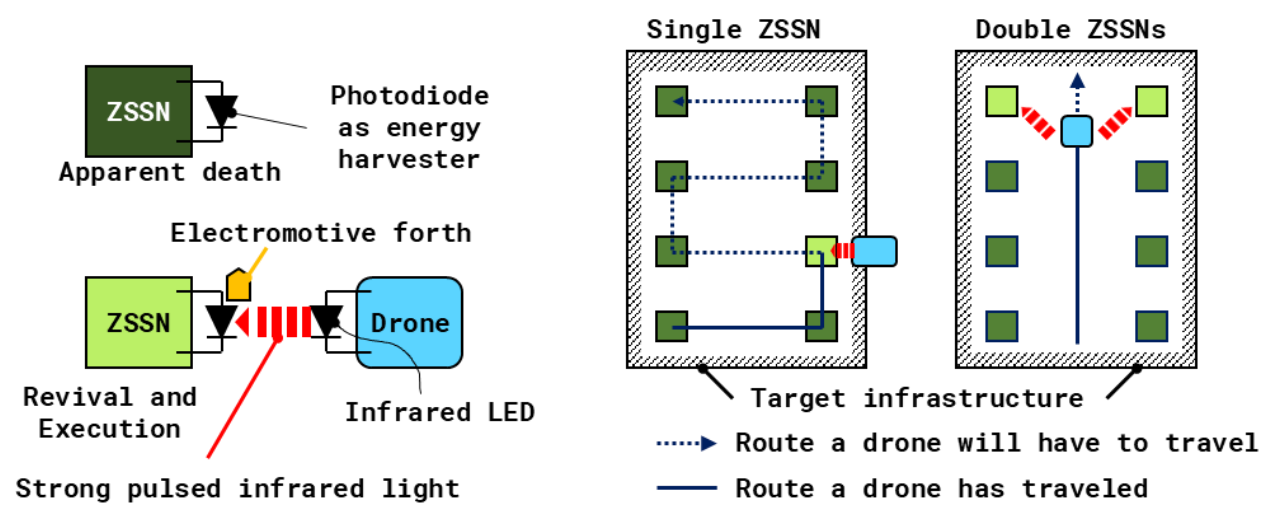

Fig. 3 Infrastructure Monitoring using ZSSN

\subsection{Infrastructure Monitoring System using ZSSN}

To realize an efficient infrastructure monitoring system, we have proposed an infrastructure monitoring system combination of many ZSSNs and a drone as shown in Fig.3. The ZSSN has a photodiode with a sensitivity to an infrared light as an energy harvester shown in Fig. 1. The drone has an infrared LED with a strong iradiating performance. The drone irradiates the strong pulsed infrared light to wake the ZSSN up. The photodiode on the ZSSN generates some electromotive forth when receiving the infrared light. This electromotive forth must be enough strong and long so as to wake the ZSSN up completely.

The ZSSNs with the number required are attached to the target infrastructure. The drone has to travel all ZSSNs along the route previously set if the drone wakes single ZSSN up. In contrast, if the drone can wake the multiple ZSSNs up, the route of the drone traveling may become simple and short. As a result. the investigation of the infrastructure can be completed faster. The battery of drone may be used longer. This fact may lead to greatly reduce the burden caused by monitoring the infrastructure.

\subsection{Research Objective}

The battery consumption of ZSSN however may become larger when multiple ZSSNs are awakened simultaneously. This is because the target multiple ZSSNs must remain running until the drone finishes gathering all sensed data from them. The time of gathering sensed data from multiple ZSSNs will become longer than that of single ZSSN.

The battery life of ZSSN is dependent on the execution time but not the apparent death time ${ }^{(3)}$. Previously, we shown the battery life of the ZSSN is enough large for practical applications compared with the conventional sensor node ${ }^{(3)}$. This proof may be broken when the strategy

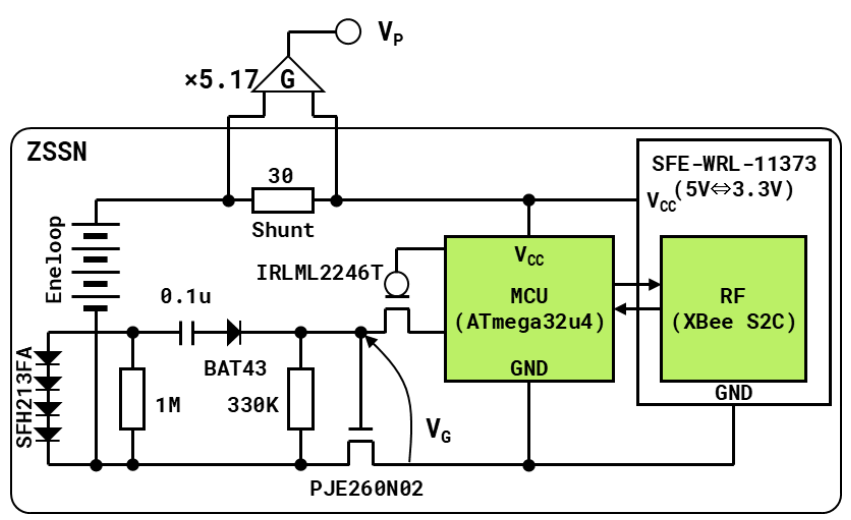

Fig. 4 Block Diagram of Prototype ZSSN

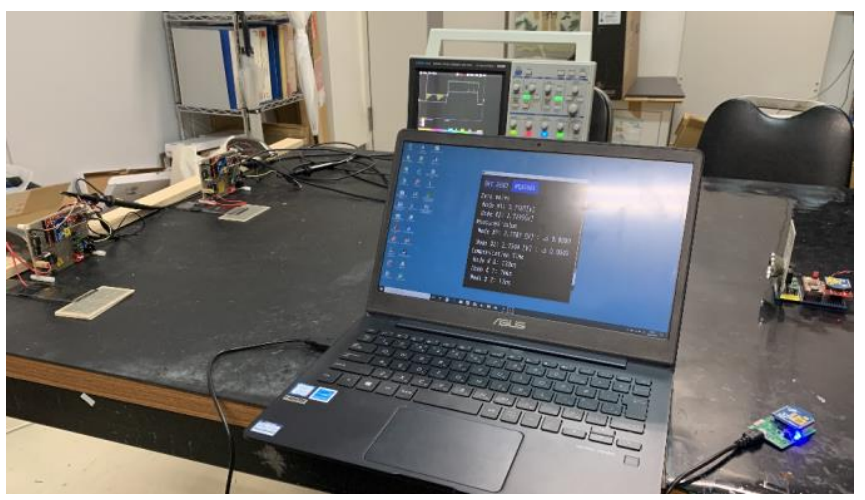

Fig. 5 Picture of Experimental Setup

awaking multiple ZSSNs simultaneously to reduce the burden of the drone gathering is taken.

Thus, this paper investigates the tradeoff between the battery life and the number of ZSSNs awaken at same time by using the measured parameters on a prototype hardware.

\section{Experimental Setup}

To estimate the battery life of ZSSN, we have to measure some parameters on a prototype hardware ${ }^{(3)}$. Fig. 4 is the block diagram of the prototype hardware we have developed. The parameters needed are the execution time 

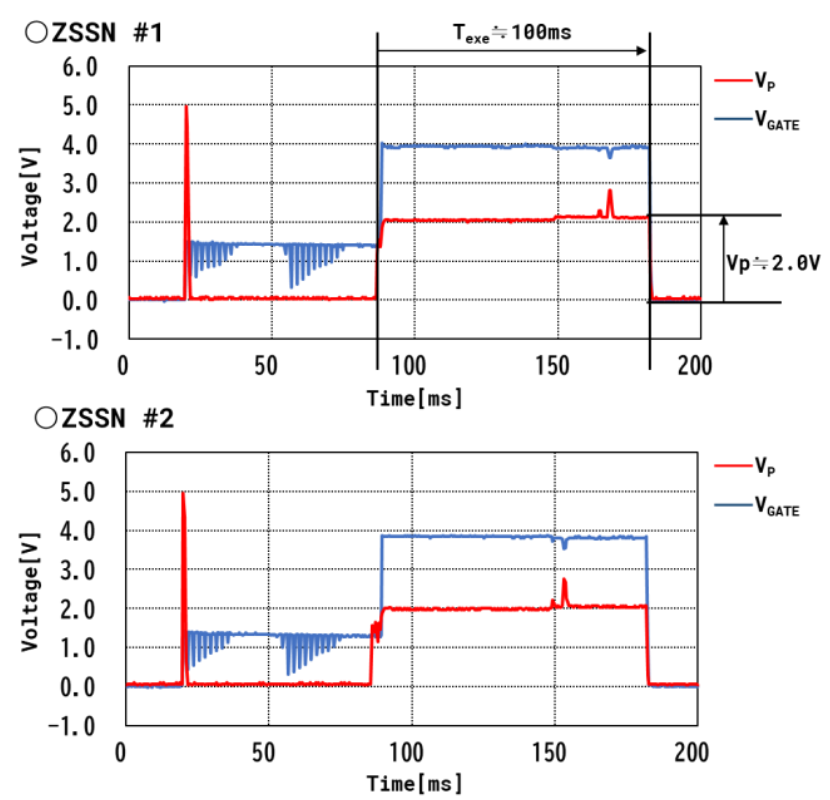

Fig. 6 Measured Waveform of Prototype ZSSNs

and the consumed current when the ZSSN is running. The execution time was measured by observing the waveform of the gate signal to the n-type transistor bridging the ground line, $\mathrm{V}_{\mathrm{G}}$. The consumed current in running was measured by observing the voltage over the shunt register, $\mathrm{V}_{\mathrm{P}}$. Fig. 5 is the picture of the experimental setup. We developed and used two same ZSSNs.

\section{Experimental Result and Discussion}

The battery life of the ZSSN can be estimated by using Eq. (1) ${ }^{(3)}$.

$$
B L=\frac{8.38 \times B C[\mathrm{Ah}]}{I_{\text {exe }}[\mathrm{A}] \times T_{\text {exe }}[\mathrm{s}] \times N} \text { [year] }
$$

The $B L$ is the battery life of ZSSN whose unit is year. The $B C$ is the capacity of battery multiplied by the derating factor of $85 \%$ whose unit is Ah. We set the $B C$ to 2000 $[\mathrm{mAh}] \times 0.85=1.7[\mathrm{Ah}]$. The $I_{\text {exe }}$ and $T_{\text {exe }}$ are the consumed current and execution time while the ZSSN is running. Their unit is second. The $N$ is the number of revivals per day.

Fig. 6 shows the measured result. The $T_{\text {exe }}$ is about 100 [ms]. The Vp is about 2.0 [V]. Thus, the consumed current when the ZSSN is running, $I_{\text {exe }}$, is $2.0[\mathrm{~V}] / 5.17 / 30[\Omega]=$ $13[\mathrm{~mA}]$.

These results are the case of two ZSSNs. We measured the time extended by adding one ZSSN is 16 [ms] as shown in Fig. 7. Given the number of ZSSNs as NN, Eq. (1) can be modified to Eq. (2) in order to consider the time

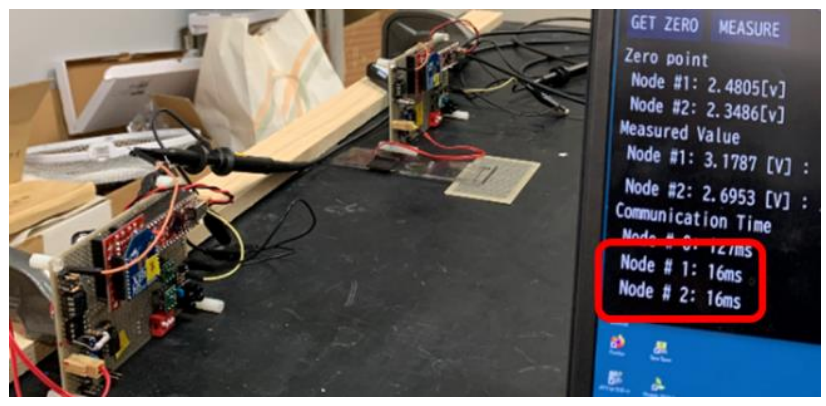

Fig. 7 Measured Time Extended by Adding One ZSSN extended every addition of the ZSSN.

$$
B L^{\prime}=\frac{8.38 \times 1.7[\mathrm{Ah}]}{13 \times 10^{-3}[\mathrm{~A}] \times\{84+(N N-1) \times 16\} \times 10^{-3}[\mathrm{~s}] \times N} \text { [year] }
$$

Fig. 8 shows the estimated result by using Eq. (2) changing the number of revivals per day, $N$, and the number of ZSSNs, NN.

How many ZSSNs can be awaken simultaneously is dependent on the performance and number of infrared LEDs attached to the drone. Thus, we think that the range of NN from 1 to 16 shown in Fig. 8 may be reasonable number. As expected, the battery life becomes shorter as the number of ZSSNs simultaneously awaken becomes larger. However, the battery life is longer than 100 years when the number of investigations to the target infrastructure is lower than 20 regardless of the number of ZSSNs simultaneously awaken. This estimation would indicate that many infrastructure investigations can accept the usefulness of ZSSNs.

\section{Conclusions}

To realize an efficient infrastructure monitoring system, we have proposed combination of zero standby power-type sensor node (ZSSN) and UAV like drone.

Since the ZSSNs wake up from the state power supply

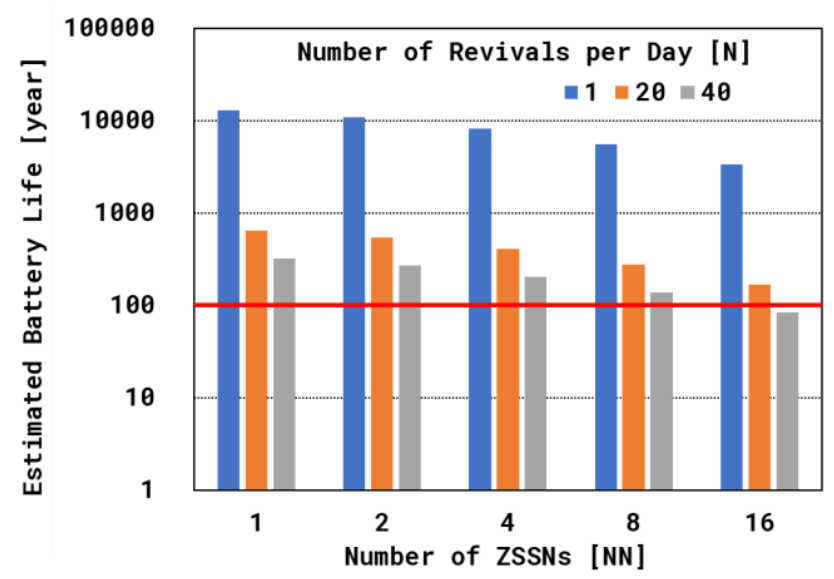

Fig. 8 Estimated Battery Life of ZSSNs 
is completely cut, the waking time may be longer. As a result, the traveling time of UAV also becomes longer. This may lead to shorten the battery life of UAV and to enlarge the maintenance cost after all.

This paper investigates the gathering time from multiple ZSSNs simultaneously awaken and discusses how much reduce the traveling cost of UAV by eliminating the necessity to visit all ZSSNs.

Estimated result indicates that to simultaneously awake reasonable number of ZSSNs can reduce the burden to the infrastructure investigation greatly without bad affection to the battery life of ZSSNs.

As future work, we will develop the real applications using ZSSNs for the infrastructures. Then, we will evaluate the usefulness of ZSSNs practically.

\section{Acknowledgment}

This work was supported by JSPS KAKENHI Grant Number 17K00126.

\section{References}

(1) Yuta Suzuki, Yuichi Morita and Akira Yamawaki: "An Investigation to Realize Zero Standby Strain Sensor Node", Proceedings of the 33rd International Technical Conference on Circuits/Systems, Computers and Communications (ITC-CSCC) 2018, pp.70-73 (2018.7)

(2) Yuta Suzuki, Shogo Yoshikawa, and Akira Yamawaki: "A Command List Based Automatic Controlling Method of UAV for Infrastructure Monitoring System with Zero-standby Sensor Node", Proceedings of the 6 the IIAE International Conference on Industrial Application Engineering 2018, pp.215-218 (2018.3)

(3) Akira Yamawaki and Seiichi Serikawa: "Battery Life Estimation of Sensor Node with Zero Standby Power Consumption", Proceedings of 19th IEEE International Conference on Computational Science and Engineering, pp.166-172 (2016.8)

(4) Akira Yamawaki and Seiichi Serikawa: "Door Monitoring System Using Sensor Node with Zero Standby Power", Transactions on Engineering Technologies: International MultiConference of Engineers and Computer Scientists 2016, pp.73-87 (2017.5)

(5) Akira Yamawaki, Mayu Yamanaka and Seiichi Serikawa: "A sensor node architecture with zero standby power on wireless sensor network", Artificial
Life and Robotics, vol. 20, no. 3, pp.210-216 (2015.10) 